\section{Blue rubber bleb nevus syndrome: combined surgical and endoscopic treatment}

Blue rubber bleb nevus syndrome (BRBNS) is a rare disease associated with multiple venous malformations in the skin, gastrointestinal tract, and other organs [1], which usually presents with extensive hemorrhage or anemia [2]. Different therapeutic strategies have been proposed.

We describe a case of BRBNS involving the small bowel, which was treated using a combined surgical and endoscopic approach. A 34-year-old woman with a diagnosis of BRBNS and a history of severe iron deficiency anemia was admitted for a small-bowel capsule endoscopy, which showed multiple vascular nodular lesions with signs of recent bleeding in the jejunum and proximal ileum ( $\bullet$ Fig.1). The patient was treated with blood transfusions and iron supplementation. A double-balloon enteroscopy was performed 2 months later because of new episodes of severe anemia ( $\bullet$ Fig. 2 ), and visible proximal and distal vascular malformations were marked with ink ( $\bullet$ Fig.3). Computed tomography enterography revealed multiple vascular malformations also in the distal tract of the small bowel. Finally, we used a rendezvous approach with surgery and intraoperative enteroscopy.

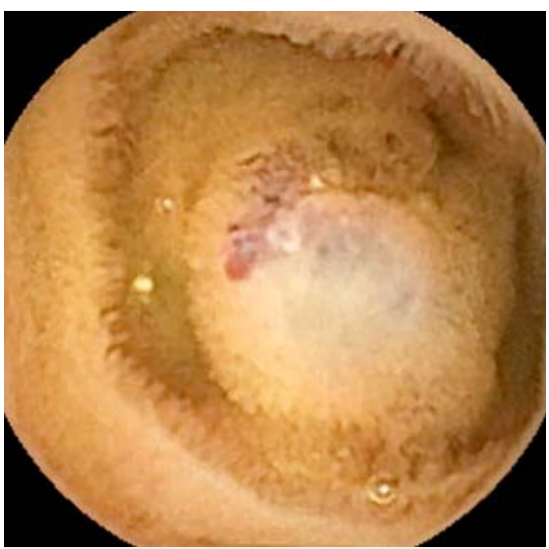

Fig. 1 Small-bowel capsule endoscopy showing multiple blue vascular nodular lesions in the jejunum and proximal ileum of a 34-year-old woman with blue rubber bleb nevus syndrome (BRBNS), who was sufferingfrom frequent episodes of severe anemia.
During the laparotomy, the parts of the ileal tracts with the majority of vascular lesions were identified. Two enterotomies were then performed, through which an endoscope was introduced to allow elastic band ligation to be carried out, whether upstream or downstream, of the remaining vascular lesions ( $\bullet$ Fig. 4a,b). Surgical segmental resection of the two previously identified ileal tracts was performed ( $\bullet$ Fig.5). The postoperative course was normal. A follow-up small-bowel capsule endoscopy showed only three jejunal and two ileal vascular lesions, which were not bleeding. The patient remains in good clinical condition and has had no need for further blood transfusions.

The treatment of BRBNS is mainly conservative. Antiangiogenic agents [3], endoscopic therapy (laser photocoagulation, sclerosis, band ligation, and polypectomy) [4], and surgery [5] have been utilized to reduce bleeding episodes. Laparotomy with intraoperative endoscopy could be used as an alternative approach in selected patients with refractory anemia and multiple lesions in the gastrointestinal tract.

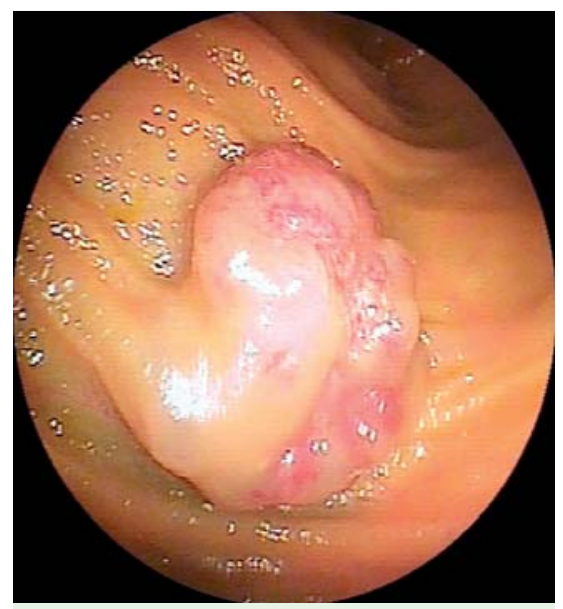

Fig. 2 Double-balloon enteroscopy showed vascular lesions with signs of recent bleeding.

\section{Competing interests: None}

\section{Monica Arena', Matteo Virdis², Elisabetta Morandi ${ }^{1}$, Paolo Viaggi ${ }^{1}$, Andrea Pisani², Enrico Opocher ${ }^{2}$, Enzo $\mathrm{Masci}^{3}$}

${ }^{1}$ Department of Gastrointestinal Endoscopy, San Paolo University Hospital, Milan, Italy

2 Department of Surgery, San Paolo University Hospital, University of Milan, Milan, Italy

${ }^{3}$ Gastrointestinal Endoscopy, IRCCS National Cancer Institute, Milan, Italy

\section{References}

1 Bean WB. Blue rubber-bleb nevi of the skin and gastrointestinal tract. In: Bean WB. Vascular spiders and related lesions of the skin. Springfield, Illinois: Charles C Thomas; 1958: $17-185$

2 Dwivedi M, Misra SP. Blue rubber bleb nevus syndrome causing upper GI hemorrhage: a novel management approach and review. Gastrointest Endosc 2002; 55: 943-946

3 Gonzalez D, Elizondo BJ, Haslag $S$ et al. Chronic subcutaneous octreotide decreases gastrointestinal blood loss in blue rubberbleb nevus syndrome. J Pediatr Gastroenterol Nutr 2001; 33: 183-188

4 Guo W, Peng Z, Tang X et al. Endoscopic management of blue rubber bleb nevus syndrome: A case report. Exp Ther Med 2013; 6: $1159-1162$

5 Choi KK, Kim JY, Kim MJ et al. Radical resection of intestinal blue rubber bleb nevus syndrome. J Korean Surg Soc 2012; 83: 316-320

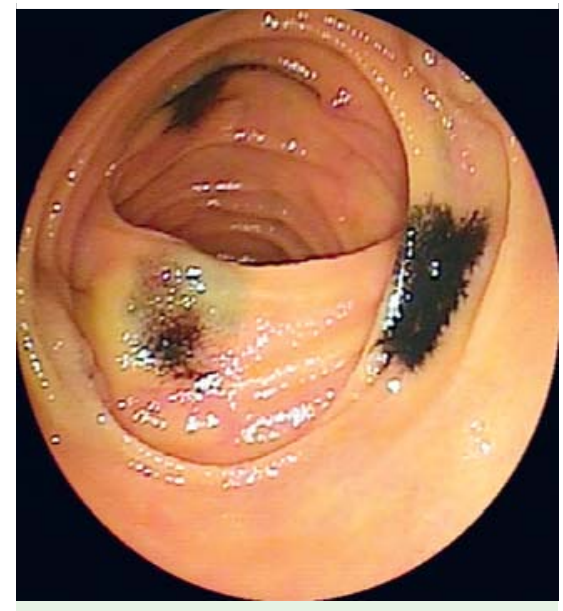

Fig. 3 Proximal and distal visible vascular malformations were marked with ink. 


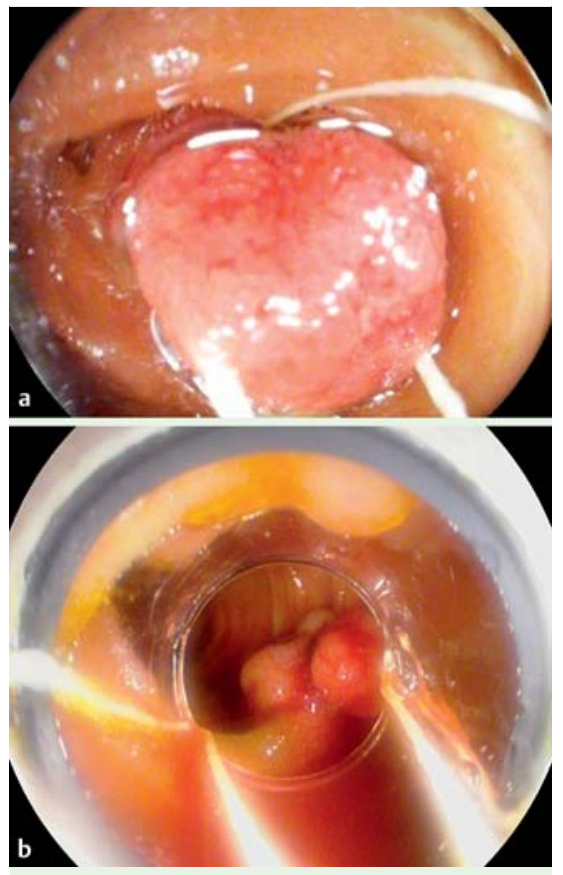

Fig. 4 a Intraoperative enteroscopy with endoscopic elastic band ligation of vascular lesions in a case of blue rubber bleb nevus syndrome. $\mathbf{b}$ Elastic band ligation of a vascular lesion.

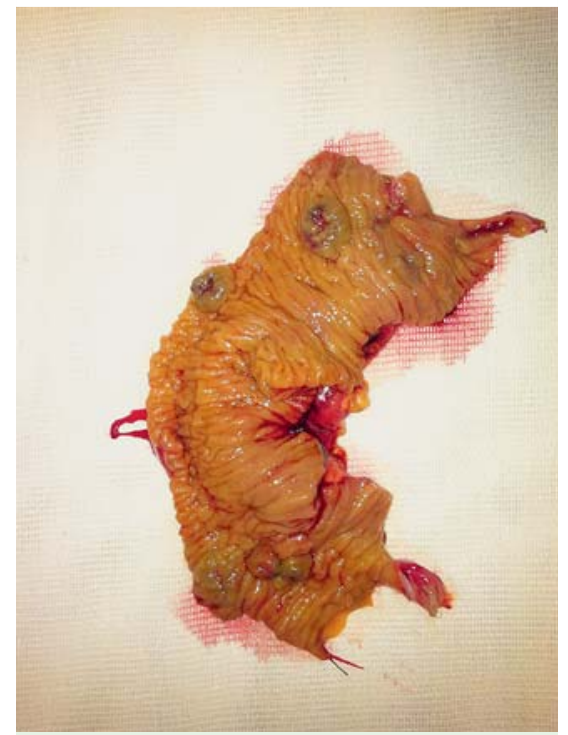

Bibliography

DOI http://dx.doi.org/

10.1055/s-0034-1392635

Endoscopy 2015; 47: E372-E373

(c) Georg Thieme Verlag KG

Stuttgart · New York

ISSN 0013-726X

\section{Corresponding author}

Monica Arena, MD

Department of Gastrointestinal Endoscopy San Paolo University Hospital

Via A. di Rudinì 8

20142, Milano

Italy

Fax: +3902 81844467

m_arena9@virgilio.it

Fig. 5 Specimen from surgical segmental resection of the ileal tract following enteroscopy with endoscopic ligation of vascular lesions. 